\title{
BMJ Open Emergency supply of prescription-only medicines to patients by community pharmacists: a mixed methods evaluation incorporating patient, pharmacist and GP perspectives
}

Charles W Morecroft, ${ }^{1}$ Adam J Mackridge, ${ }^{1}$ Elizabeth C Stokes, ${ }^{2}$ Nicola J Gray, ${ }^{3}$
Sarah E Wilson, ${ }^{4}$ Darren M Ashcroft, ${ }^{5}$ Noah Mensah, ${ }^{6}$ Graham B Pickup ${ }^{7}$

\section{ABSTRACT}

Objective: To evaluate and inform emergency supply of prescription-only medicines by community pharmacists (CPs), including how the service could form an integral component of established healthcare provision to maximise adherence.

Design: Mixed methods. 4 phases: prospective audit of emergency supply requests for prescribed medicines (October-November 2012 and April 2013); interviews with CPs (February-April 2013); follow-up interviews with patients (April-May 2013); interactive feedback sessions with general practice teams (OctoberNovember 2013).

- Prepublication history and additional material is available. To view please visit the journal (http://dx.doi.org/ 10.1136/bmjopen-2014006934).

Received 16 October 2014 Revised 13 May 2015 Accepted 22 May 2015

CrossMark

For numbered affiliations see end of article.

Correspondence to

Professor Charles W Morecroft; C.W.Morecroft@ljmu.ac.uk
Setting: 22 community pharmacies and 6 general practices in Northwest England.

Participants: 27 CPs with experience of dealing with requests for emergency supplies; 25 patients who received an emergency supply of a prescribed medicine; 58 staff at 6 general practices.

Results: Clinical audit in 22 pharmacies over two 4-week periods reported that 526 medicines were requested by 450 patients. Requests peaked over a bank holiday and around weekends. A significant number of supplies were made during practice opening hours. Most requests were for older patients and for medicines used in long-term conditions. Difficulty in renewing repeat medication (forgetting to order, or prescription delays) was the major reason for requests. The majority of medicines were 'loaned' in advance of a National Health Service (NHS) prescription. Interviews with CPs and patients indicated that continuous supply had a positive impact on medicines adherence, removing the need to access urgent care.

General practice staff were surprised and concerned by the extent of emergency supply episodes.

Conclusions: CPs regularly provide emergency supplies to patients who run out of their repeat medication, including during practice opening hours. This may aid adherence. There is currently no feedback loop, however, to general practice. Patient care and interprofessional communication may be better served by the introduction of a formally structured and funded NHS emergency supply service from community

\section{Strengths and limitations of this study}

- This paper expands the sparse literature about the occurrence and characteristics of emergency supplies of prescription-only medicines made through community pharmacies to patients.

- This paper examines the perspectives of the three major stakeholder groups-patients, pharmacists and general practice staff.

- The participants were self-selected and this may introduce bias.

- The number of participants in each stakeholder group was modest.

- The study has generated useful underpinning information for further practice and policy development in this field.

pharmacies, with ongoing optimisation of repeat prescribing.

\section{INTRODUCTION}

The Medicines Act 1968, and latterly the Human Medicines Regulations 2012, permit community pharmacists (CPs) to supply prescription-only medicines (POMs) without a prescription in an emergency when requested by either a prescriber or the patient. ${ }^{1}$ This enables pharmacists to use their professional judgement to ensure continuous supply of medicines. Pharmacists 'must be satisfied' that there is an 'immediate need' for the medicine, while also considering the patient's well-being and any consequences of not supplying (box 1). ${ }^{2}$ In this paper, the term 'emergency supply' refers to both the supply of medicines where a charge is made directly to the patient, and to the 'loan' of medication made by CPs where no 
Interview. the pharmacist must interview the patient, preferably face to face

Immediate need: the pharmacist must be satisfied that there is an immediate need for the prescription-only medicine (POM) and that it is not practical for the patient to obtain a prescription without undue delay

Previous treatment. the POM requested must previously have been used as a treatment and prescribed by a relevant prescriber

Dose: the pharmacist must be satisfied of knowing the dose that the patient needs to take

Not for controlled drugs, except phenobarbital: medicinal products cannot be supplied if they consist of or contain any schedule 1,2 or 3 controlled drugs; phenobarbital can be supplied to patients of UK-registered prescribers for the purpose of treating epilepsy

Length of treatment. if the emergency supply is for a controlled drug (ie, phenobarbital or schedule 4 or 5 controlled drug), the maximum quantity that can be supplied is for 5 days' treatment. For any other POM, no more than 30 days can be supplied except:

- If the POM is insulin, an ointment, a cream or an inhaler for asthma (ie, the packs cannot be broken), the smallest pack available in the pharmacy should be supplied;

- If the POM is an oral contraceptive, a full treatment cycle should be supplied;

- If the POM is an antibiotic in liquid form for oral administration, the smallest quantity that will provide a full course of treatment should be supplied.

Records kept. an entry must be made in the POM register on the day of the supply (or, if impractical, on the following day). The entry needs to include: date supplied; name (including strength and form where appropriate) and quantity of medicine supplied; name and address of patient; and information on nature of emergency

Labelling: in addition to standard labelling requirements, the words 'Emergency Supply' need to be added to the dispensing label

charge is made to the patient and the supply is subsequently reconciled against a future National Health Service (NHS) prescription. Emergency supplies may also be made at the request of a prescriber, but those are not included in this paper. For loans in anticipation of a future NHS prescription, the additional work (ie, clinical check, determining evidence of previous supply, dispensing and documentation) undertaken by the pharmacists is not remunerated, either by the patient or the NHS. For an emergency supply where a future NHS prescription is unlikely to be obtained, usually for outside visitors to the locality, a charge is made to the patient to cover the medicine costs, and a discretionary small amount for administration.

Dispensing services around the world are likely to identify with the issues of emergency supply or owing/loaning medication outlined above. Where prescription medicine supply is tightly regulated-like in the UK, the USA, Europe, Canada and Australasia-an emergency supply service similar to the England version prevails. In countries where more prescription medicines are sold in pharmacies, like the Middle and Far East, patients are able to purchase these medicines directly, although evidence of previous use (such as old medicine packets) may be required. There are also some countries where no provision for pharmacy emergency supply exists and where the patient would have to visit a doctor for a prescription.

A 1998 survey of CPs by O'Neill et $a \hat{l}$ demonstrated that requests for emergency supplies and medicine loans were being made on a frequent basis: at least monthly for three-quarters of respondents, at least weekly for half, and at least daily for 1 in 10 . Respondents perceived emergency supplies as an important service, but over three-quarters felt it was open to misuse. Other studies in this field suggest variability in professional decision-making processes and justifications, and there remains no clear definition as to what constitutes 'immediate need' ${ }^{4-8}$ While pharmacists identify themselves as acting in the patient's best interests, previous studies have not explored what impact supply or refusal might have on patient adherence to treatment. A significant number of emergency medicine requests are being made to urgent care services. Urgent Care 24 (UC24), a local provider of general practitioner (GP) out-of-hours services in Liverpool, reported that a total of 5156 repeat medication requests out-of-hours were received by the service in the period of September 2012September $2013,{ }^{9}$ for a patient population of approximately 750000.

NHS England's Call to Action highlighted challenges regarding more patients with long-term conditions and increasing patient expectations. ${ }^{10} \mathrm{CPs}$ are named among healthcare professionals who can support patients in managing long-term conditions in primary care. A Royal Pharmaceutical Society (RPS) report on future models of care for pharmacy ${ }^{11}$ highlights the potential of community pharmacy in GP out-of-hours services and urgent care. Joint work between NHS England and the Pharmaceutical Services Negotiating Committee has explored extending pharmacy services to relieve pressure on accident and emergency (A\&E) departments while maintaining standards of care, including amendments which permit NHS England Area Teams (regional commissioners) to commission 'Emergency Supply at NHS expense' as an Enhanced Service from community pharmacies. ${ }^{12-14}$ A review of the literature has identified only a few studies that have explored emergency supplies by community pharmacies in depth; the majority were based in the UK. ${ }^{3-8}$ Moreover, there is a need for a holistic examination, incorporating multiple viewpoints, of how the emergency supply service at community pharmacies may best fit within current and established health and social care provision in order to best support patient care. This evaluation aims to describe the current profile of emergency supply activity in community pharmacies to explore and inform future practice. 


\section{METHODS}

This study used a mixed methods approach over four consecutive phases. Data collection was undertaken by practising CPs in North West England alongside the research team. Participants in all phases gave informed consent before taking part. An overview of the multiphased study is provided in table 1 (Research Protocolv1).

Phase 1: Clinical audit data of emergency supplies in participating pharmacies across North West England, over two 4-week collection periods. Pharmacies were purposively sampled via a recruitment pack, containing study information and consent forms, posted to them by the local Primary Care Research Network and Liverpool John Moores University (LJMU). Through this process, diversity in pharmacy ownership (independent vs corporate), setting and opening hours-and pharmacist gender and practice experience-was maximised. CPs in the participating pharmacies were asked to document the characteristics of all emergency supplies of prescribed medicines over two 4-week periods (October/November 2012 and April 2013). A pad of preprinted paper audit forms was supplied to each pharmacy. The form was created, piloted and validated by the practising CPs on the research team (GBP and NM) (phase 1-data capture form-1 October 2012.pdf (v1.0)—see online supplementary file). Details captured for each episode included: the day/date of the request; patient age and postcode; the quantity, name, dosage form and dose given of the medicine; the reason for the request, and whether the supply was made. No identifiable information was recorded on those forms.

Phase 2: Semistructured telephone interviews with CPs working at pharmacies across North West England. A subgroup of specially trained CPs who volunteered from the phase 1 audit cohort interviewed the other pharmacists, who were based in pharmacies with diverse locations,

\section{Table 1 Overview of study phases}

\section{Project phases}

Phase 1:

Clinical audit of emergency supplies in participating

pharmacies over two 4-week collection periods

October-November 2012 and April 2013

Phase 2:

Semistructured telephone interviews with pharmacists

working at pharmacies across North West England

February-April 2013

Phase 3:

Follow-up telephone interviews with service users who received emergency supplies/loans of prescription-only medicines

April-May 2013

Phase 4:

Qualitative interactive feedback sessions with medical

practice teams

October-November 2013 settings, opening hours and ownership type. Pharmacies who had participated in the phase 1 audit were invited to take part, and other pharmacists were recruited via local professional networks. Recruitment packs containing information and consent forms were provided to potential participants ( phase 2-participant information sheet-1 Oct 2012.pdf (v1.0)—see online supplementary file). Those who returned a completed consent form were interviewed. These telephone interviews (undertaken February/April 2013) explored pharmacists' experiences and opinions in relation to requests for emergency supplies and loans (phase 2-interview schedule-1 Oct 2012.pdf (v1.0)—see online supplementary file). It also explored their reflections on challenges encountered and their resolution strategies. Peer-to-peer interviewing facilitated effective probing of responses to elicit details of difficult situations through shared professional insight into the dilemmas described. This technique has been utilised previously during interviews conducted by GPs with fellow practitioners ${ }^{15}$ these respondents recognised the interviewer as a fellow clinician, resulting in broader and more personal accounts of their attitudes and behaviour in clinical practice. Interviews were audio recorded with consent from interviewees.

Phase 3: Telephone interviews undertaken by the research assistant (ECS) with patients who received emergency supplies/ loans of POMs. Over 6 weeks in April/May 2013, patients requesting emergency supplies or loans of POMs at participating pharmacies were invited to participate in a follow-up telephone interview after using the service. At the end of the supply, they were given a recruitment pack by the pharmacist and replied direct to the research assistant (RA) (phase 3-participant information sheet-28 November 2012.pdf (v3.0)—see online supplementary file). No demographic data were collected. These semistructured telephone interviews explored patients' views, experiences and prior knowledge of the service, as well as the perceived impact of the emergency supply on the continuity of their medicines' supply and adherence (phase 3-interview schedule-1 Oct 2012.pdf (v1.0)—see online supplementary file). Interviews were completed within 2 weeks of the initial request and were audiorecorded with patient consent and transcribed verbatim.

Phase 4: Qualitative interactive feedback sessions with medical practice teams. A subgroup of CPs volunteered from the phase 1 cohort to do this work and received further training from the research team and, with support from the RA, presented interim findings from phases 1-3 to their local general practice team. Sessions took place in October/November 2013, and explored practice staff's views and experiences regarding the emergency supply service and its impact on, and relevance to, their workflow and patient well-being (phase 4 - practice staff feedback session discussion schedule.pdf (v1.0)—see online supplementary file). Practice staff provided written informed consent to take part in the 
session (phase 4-participant information sheet-1 Oct 2012.pdf (v1.0)—see online supplementary file). To protect patient anonymity, CPs began sessions by explaining that the findings were from multiple study sites across North West England and incorporated many patients who were not registered at that practice. Field notes were taken during the discussions by the RA and consent to participate was obtained from all attendees.

\section{Data analysis}

Data were entered from the forms into IBM SPSS V.21 statistical software, where it was subjected to basic descriptive analysis. Qualitative data from all other phases were transcribed verbatim and thematically analysed for emergent themes, using NVivo V.10 software (QSR International). A 'directed content analysis' approach was used. ${ }^{16}$ Primary attention was directed at identifying broad categories of data, followed by specific line-by-line categorisation. The study objectives provided a clear source of categories with which to organise participants' responses, while allowing other themes to emerge. Analysis examined commonalities between participants as well as contrasting perceptions of the emergency supply process. Members of the project team, and the CPs who conducted the interviews, further reviewed emergent themes to ensure robustness regarding coding and reconstruction.

Data from all phases were then triangulated to provide an understanding of the service from multiple perspectives, enhancing the validity and reliability of the study outcomes.

\section{RESULTS}

\section{Participants in each phase of the project}

Phase 1 audit: Twenty-two pharmacies took part in the phase 1 audit (table 2). Diversity in pharmacist experience, gender and length of time since registration was seen. Sites were most frequently located in small parades of neighbourhood shops $(9 / 22)$ or within or adjacent to the health centre $(8 / 22)$ (table 2). Fourteen pharmacies $(63.6 \%)$ were closed at weekends, and three $(13.6 \%)$ opened for $100 \mathrm{~h}$ over 7 days.

Phase 2 CP interviews: Following training in telephone interview techniques by the research team, five pharmacist researchers (PRs) completed recorded semistructured peer telephone interviews with 26 CPs working at pharmacies across North West England. Interviewees were based in pharmacies with diverse locations, settings, opening hours and ownership type, that is, independent, small/medium chain and nationwide multiple. Nineteen of the pharmacists interviewed had been involved in phase 1 of the study with the remainder being directly recruited by the PRs via professional networks. Interviews lasted between 7 and 36 min (mean 14 min).

Phase 3 patient interviews: From the 191 recruitment packs distributed by 22 pharmacies, 30 responses were received from patients at 9 pharmacies (16\% response rate). Semistructured interviews were completed with 25 patients (2 declined to take part when contacted and 3 could not be contacted). Interviews lasted between 3 and 9 min (mean 5 min).

Phase 4 general practice feedback sessions: Fourteen general practice teams were invited to take part in this phase, of which six agreed. Reasons given for nonparticipation included: introduction of Electronic Prescription Service (EPS) occupying staff time; and a policy of refusing meetings with external parties. In some cases, practice teams appeared comfortable with meeting to hear the study findings, but were reticent about their opinions being captured. The length of time made available for the meeting varied between practices; some added the discussion to their monthly staff meeting agenda and others arranged a separate, fulllength discussion. Different general practice staff categories were represented at the feedback sessions, which took place in October/November 2013. They included 5 practice managers; 25 GPs; 12 practice nurses; 10

Table 2 Characteristics of participating pharmacies

\begin{tabular}{|c|c|c|}
\hline Characteristic & Number of pharmacies & $\begin{array}{l}\text { Percentage of } \\
\text { total pharmacies }(n=22)\end{array}$ \\
\hline \multicolumn{3}{|l|}{ Type of pharmacy ownership } \\
\hline Single independent pharmacy & 4 & 18.2 \\
\hline Small group of 2-5 pharmacies & 3 & 13.6 \\
\hline Local group of more than 5 pharmacies & 11 & 50.0 \\
\hline National group of over 100 pharmacies & 4 & 18.2 \\
\hline \multicolumn{3}{|l|}{ Location of pharmacy } \\
\hline Local parade of shops & 9 & 40.5 \\
\hline Health centre & 8 & 36.4 \\
\hline Town centre/high street & 3 & 13.6 \\
\hline Other & 2 & 9.5 \\
\hline \multicolumn{3}{|l|}{ Standard days open } \\
\hline Monday-Friday & 14 & 63.6 \\
\hline Monday-Saturday & 5 & 22.8 \\
\hline Monday-Sunday & 3 & 13.6 \\
\hline
\end{tabular}


reception and administration staff; 2 healthcare assistants; 2 district nurses; 1 phlebotomist; and 1 health visitor. Attendance ranged from 2 (the lead GP partner and practice manager) at one surgery to 17 team members at another. The duration of the six sessions ranged from 18 to $62 \mathrm{~min}$ (mean $36 \mathrm{~min}$ ).

The results of the study are presented in an integrated approach by theme, across methods.

\section{Frequency and characteristics of emergency supplies}

Emergency supply requests were made for a total of 526 medicine items by 450 patients at 22 community pharmacies over the two 4-week audit collection periods. Most requests were for single items (405/450 occasions; $90 \%$ ) with three or more items requested on 17 occasions $(4 \%)$.

A higher proportion of requests were recorded on either side of the weekend (Mondays and Fridays) than on other days, with around a quarter of items requested $(123 / 526 ; 23 \%)$ on a Friday (figure 1). In the eight pharmacies open during weekends ( 3 open both days; 2 all day Saturday; and 3 Saturday morning only), emergency supply requests were made for 65 items during this period, reflecting a higher rate per pharmacy in comparison to any of the weekdays.

Almost two-thirds (16/26) of CPs interviewed in phase 2 reported normally receiving requests at least daily, with four describing multiple requests per day, although requests rates were often variable. At phase 4 feedback sessions, some participants, including GPs and practice managers, were surprised to find that requests were received across the week; they regarded emergency supplies as something that should only happen outside practice opening times.

Emergency supply requests in phase 1 occurred for patients aged from 3 months to 92 years, with 13 (3\%) for children under the age of 12 years. Although there was a trend towards more requests from older patients, a substantial number were made by young and middleaged adults (figure 2).

Over two-thirds $(18 / 26)$ of CP interviewees in phase 2 highlighted older people as the client group most frequently requesting emergency supplies. Some respondents $(6 / 26)$ felt that this group had more difficulties in ordering their repeat prescriptions than younger people, but others $(3 / 26)$ felt that this was simply

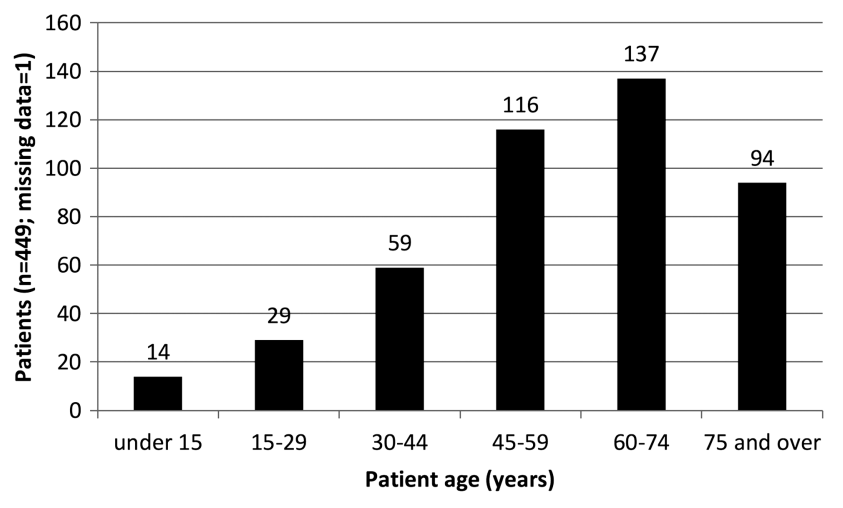

Figure 2 Distribution of emergency supply requests by patient age category $(n=449$; missing $=1)$.

related to the more frequent use of medicines in this group. Younger and middle-age groups were thought by three interviewees to be likely to request emergency supplies due to other commitments, such as working or caring for others.

Most requests in phase 1 were for medicines used in long-term conditions and the therapeutic areas broadly mirrored national prescribing profiles. ${ }^{17}$ The most commonly requested medicines were used for cardiovascular $(32 \%, 169 / 526)$, respiratory $(13 \%, 70 / 526)$, endocrine $(12 \%, 63 / 452)$ and gastrointestinal conditions (11\%, $56 / 526)$. Specific medicines that might lead to a risk of adverse clinical implications if a supply was not provided included treatment for a renal transplant (azathioprine) and cancer (letrozole). The wide range of medicines involved was confirmed by CPs in phase 2, with most stating that the majority of requests were for medicines for long-term conditions.

\section{Reasons for requests}

Difficulties associated with renewing repeat medication were the major reason recorded in phase 1 audit for emergency requests, including patients having 'forgotten to order' $(364 / 526 ; 69 \%)$, delays with prescriptions being issued $(16 / 526 ; 3 \%)$; ordered items missed off prescriptions $(14 / 526 ; 3 \%)$; and errors in ordering repeat supplies via the pharmacy, for example, incorrect strength $(8 / 526 ; 2 \%)$. Patient interviewees in phase 3 also reported repeat medication ordering as a cause for their emergency supply request, particularly at the end
Figure 1 Distribution of emergency supply requests: days of the week $(n=526)$ (POM, prescription-only medicine).

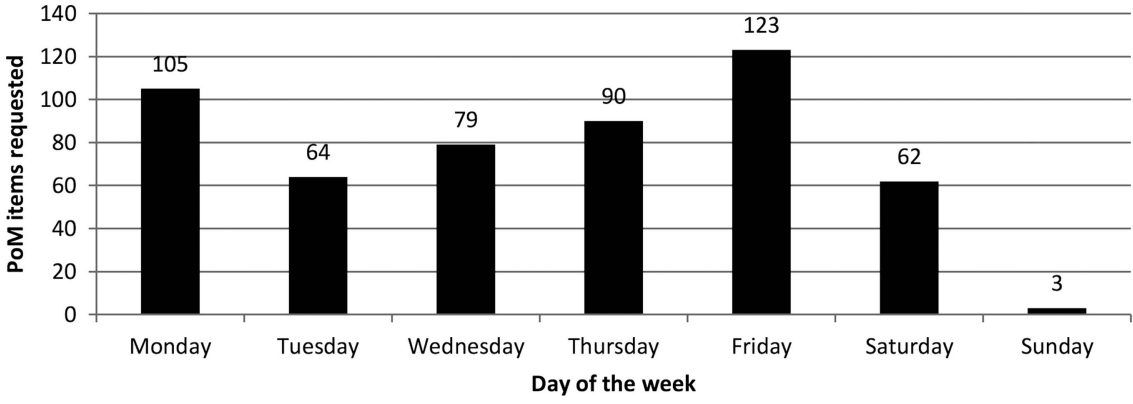


of a week where a supply was needed to cover the weekend. Some patients admitted that this was often an oversight on their part, but others mentioned life circumstances contributing to their problems with the ordering systems, including a $24 \mathrm{~h}$ carer and those who worked full-time. Other reasons for requests recorded in phase 1 were: insufficient quantities prescribed (24/526; $5 \%)$; requests following increases in the prescribed dose leading to shortages $(7 / 526 ; 1 \%)$; and prescribed quantities of medicines being out-of-sync with multiple repeat dates $(30 / 526 ; 6 \%)$. Problems with unsynchronised medicines prescribing, that is, where one or more additional medicines are started at a different time to other medicines, were also reported as the cause of the problem for five of the phase 3 patients.

\section{Responding to requests}

The majority $(489 / 526 ; 93 \%)$ of item requests in phase 1 related to medicines 'loaned' to the patient in anticipation of an NHS prescription. In the few cases (17/ $526 ; 3 \%)$ where a charge was made, this was usually because the patient was on holiday and had forgotten their medicines and it would not have been practical to obtain a prescription. In all of the emergency supplies made to the 25 phase 3 patients, medicines had been supplied as a loan, with a subsequent NHS prescription being requested by the pharmacist to claim payment.

When prompted about payment, phase 3 patients were largely unaware that the service was not a standard aspect of the NHS supply service and, in many cases, felt that they should not have to pay as they were exempt from prescription charges. In the phase 4 feedback sessions, practice staff also considered loans the more appropriate mechanism (rather than charging the patient), since the majority of requests related to repeat medication from the patient's regular pharmacy. However, one GP commented that charging patients may act as a deterrent to the patient making a future emergency supply request, suggesting that some individuals may use the loan mechanism in preference to the standard procedure.

\section{Impact on medicines adherence}

Over half of the phase 2 CPs $(15 / 26)$ described the emergency supply of medicines as a mechanism to ensure continuity of treatment as having a positive influence on adherence. Additionally, seven described it as a 'safety net' and a further two described its importance where there were delays in the processing of a prescription. Respondents also speculated that, without emergency supplies being available, some patients would simply stop their treatment until the medicines were available again.

Four of the phase 2 CPs considered the emergency supply service to have little or no impact on patient adherence. Nine expressed frustration that, although they saw the benefits in genuine emergencies, some patients abused the system, rather than managing their medicines properly. However, emergency supply requests were considered to provide opportunities to engage such patients over adherence and managing their medicines via informal discussion or through Medicines Use Reviews, an NHS-funded service for pharmacists to discuss broad issues of medicine-taking with their patients. ${ }^{18}$

Some phase 2 CPs and phase 4 GPs agreed that, while certain medicines did not need to be supplied urgently, if emergency supplies were refused in such cases, this might give patients mixed messages about the importance of adherence (box 2). In addition, some GPs highlighted that failure to supply could be interpreted as negligence if a patient were to experience an adverse health event owing to the interruption in treatment.

Phase 3 patients were asked to reflect on the impact of this emergency supply on the management of their medicines and condition. Many respondents explained that the supply had maintained their use of medicines as prescribed and one-third $(8 / 25)$ said that this gave them peace of mind with respect to their treatment. Two-thirds of patients $(16 / 25)$ emphasised the importance of an uninterrupted supply of medicines, describing the possible impact that they believed a missed dose might have (box 3). Patients also recognised that emergency supplies should not be a routine mechanism for them to obtain medicines, and one respondent described how the incident had made her more vigilant about ordering medication on time to ensure that she had a continuing supply.

Phase 3 patients described possible alternative actions that they would have taken in the absence of an emergency supply. Half $(12 / 25)$ said they would speak to their GP or the surgery receptionist in the first instance, although some were unsure whether an appointment would be possible at short notice. Using the walk-in centre, the A\&E department or GP out-of-hours service was also mentioned by four respondents.

Around a quarter of phase 3 patients $(7 / 25)$ said that they would have just done without their medicines until their prescription was ready. In some cases, participants felt that, although this would not be ideal, it would not cause any particular harm. However, others commented that this might have a negative impact on them. Four respondents described purchasing over-the-counter medicines as a possibility, although they felt that these would be less effective than their usual medication. One service user reported having previously borrowed medicines from friends (warfarin) when he ran out. Practice staff were not surprised by the alternative actions that patients described in the phase 3 interviews, including even this case of the individual who borrowed warfarin from his friend, as they recognised such behaviour from their own patients.

\section{Changes to practice}

Phase 2 CPs highlighted that the numbers of loans currently supplied were a small, but important, facet of the existing NHS supply arrangements and structuring these supplies as a funded NHS service would be helpful 
Box 2 Phase 4 practice staff quotes

\section{The challenges of emergency supply}

I think the number of steps involved it sounds easy from the outside. The patient gives in the script and he expects it to happen like that, but there are so many steps involved in it coming to the doctor and going to the pharmacist... They come at three o'clock and they want a script by five o'clock before you close. So I need to drop everything what I'm doing to do the script so it puts a lot of pressure on the service. You just need two or three people to unbalance the whole thing. (Pract1, GP)

I know from experience on a Friday everybody needs that medication because they can't wait the two days till Monday.... we know what it's like but it's hard for us to know what is urgent and, you know, what can wait till Monday. (Pract3, Receptionist)

\section{Promoting adherence}

Even though we know realistically somebody's blood pressure isn't going to shoot up and somebody's not going to suddenly have a stroke, psychologically trying to convince patients of that is very difficult...and if something adverse did happen, they would blame the fact that they didn't have the medication. It's, it's hard to get the balance right... 'You can miss it every now and then. It doesn't matter'. Yes you're sending a contradictory message...If you start saying, 'Well that doesn't matter that much', people will stop taking medication regularly or might stop it altogether. (Pract5, GP)

It's not ideal but...you can't leave the patient without any medication and that's the decision you've got to make...you're put in a difficult position but[you have to do] what's in the patient's best interests, I suppose. (Pract2, GP)

\section{Communication and relationships}

I've worked in practices where there's very often been a pharmacist like you who you get to know personally... The problem is when requests are coming for prescriptions to pay back tablets that have been lent out from a chemist that you're not really that familiar with, and we start to wonder about what the patient's up to. (Pract5, GP)

The thing is I think the problem is because they[patients] can, they can actually access you and bypass us. The whole problem stems from that. If you say everything has to come through the GP and they have to come here for the repeat prescriptions that problem doesn't arise... So those incidents should not happen. (Pract1, GP)

\section{Changes to practice}

We do get reports from Out-of-Hours services that people present at Out-of-Hours services requesting prescriptions for inhalers or blood pressure or heart medications etc. and clearly that's using out-of-hours resources which isn't appropriate. So if the pharmacist is able to do that, then it's going to save pressure on the Out-of-Hours services. (Pract6, GP)

There's a few[patients] who would misuse it so we need to identify those...I think it[feedback to GP] gives you a bit more confidence doesn't it? As a pharmacist: 'I've done this, I've let the GP know' and there's a safety net somewhere that would pick up a problem if there was an issue. I think that's not a bad idea. (Pract1, GP)

If we were informed who was using the service we could explore what were the reasons and maybe reduce that.' (Pract3, practice nurse)

I for the life of me don't understand why we have to spend so many hours a week writing prescriptions for things that people know that they should be on all the time. And I've got no understanding as to why we don't do that through pharmacies. [...] I think pharmacies would be far better at actually monitoring the number of prescriptions that have gone through. (Pract5, GP)

Ninety-nine percent of patients are taking charge or responsibility for their prescriptions; we are going after that one percent. Are we going to throw so many resources at this one per cent? (Pract1, GP)

(box 4). It was suggested that this could operate as an advanced service (nationally commissioned), such as Medicines Use Reviews, or an enhanced service (locally commissioned), such as the minor ailments service in operation in the study area. Respondents felt that such a service would need clear and transparent terms of service and associated fees to provide recognition of the additional workload for the $\mathrm{CP}$ and the expertise involved in providing the service. A national service specification was also considered important to ensure a consistent service, which would be useful for patients and other health professionals in understanding and referring to the service.

The importance of patients accessing care in the right place (GP/out-of-hours service/A\&E/Community pharmacy, etc.) for their needs was also emphasised. Pharmacists and GPs identified the role that CPs could have in removing unnecessary demand from GP out-of-hours services regarding medicines supply and that this could be a driver for change to a funded emergency supply service in community pharmacies. This provision was considered to be likely to be comparatively cost-effective, directing limited NHS resources to be used in the best way. A coordinated approach to promoting such a service was preferred, with other relevant NHS service providers having clear pathways for referral. One GP contextualised the emergency supply issue as part of the broader challenge of supplying long-term repeat prescriptions, and felt that the pharmacist might take a greater facilitating role that would reduce the need for practice staff administration. In contrast, another GP felt that emergency supplies were being requested by only a small proportion of the patient population and questioned whether the resource could be justified.

Although it was recognised that there would always be a cohort of patients who would request this type of supply, it was strongly felt that a formal service should not support patients who repeatedly forgot to order repeat prescriptions and may be considered to be abusing the system. Continued patient education at each point of access to the service, together with appropriate action by pharmacists/GPs (eg, synchronisation of 


\section{Box 3 Phase 3 patient quotes}

\section{Importance of uninterrupted supply}

She has to take it every day...she has brittle asthma and she's been in the paediatric ICU on occasion. She had been poorly and she absolutely does need it. It's vital for her. (Mother who requested emergency supply of an inhaler for her daughter aged 11)

This resident is dependent on this medication on a daily basis. It's to do with her mental health issue. It was important that we made sure that she took her medication otherwise there would be relapses. (Member of staff at supported living home for people with learning disabilities who requested emergency supply of medicines prescribed for a tenant's mental health condition)

It was on a loan because obviously I was picking my full prescription up the next day so it was just to tide me over for that one day. (Female who experienced delay in repeat prescription being forthcoming due to staff shortage at the GP's surgery)

\section{Use of urgent care services}

I probably would have had to have gone Out-of-Hours or maybe up to A\&E or drop in centre-probably explain my situation from that point of view...So yeah, it would have been far more complicated and far more awkward to be able to resolve the situation, the predicament that I was in. (Male, requested emergency supply as he had left regular medication at holiday home after weekend visit)

Well I probably would have demanded to see the doctor and then if not, I would have called the Out-of-Hours probably if I was in a mood...Depends what type of mood I'm in but I really was needing them because if I haven't had them for a few days I start getting really bad. (Female, requested emergency supply of medication to control symptoms of anxiety)

medications, review of asthma inhaler use, etc), was considered to be preferable and, over time, likely to lead to fewer patients making repeated requests. Formalised feedback to the GP about the emergency supply made might also help to improve appropriate use of the service and was supported by CP, GP and nurse participants.

\section{DISCUSSION}

Results indicate that CPs are supporting continuity of medicines use by supplying them to patients without prescription on an occasional, but routine, basis. This is particularly prevalent around times when other health services are not available, such as weekends and bank holidays, but also happens 'in-hours' during the week. Many requests are from elderly patients and individuals with long-term conditions, but all age groups are represented and a wide range of medications involved. Practice staff seemed unaware of the extent of emergency supply, especially during the week when practices were open. Practice staff acknowledged, however, that patients made requests for medication that they needed more quickly than the standard $48 \mathrm{~h}$ wait. GPs and

\section{Box 4 Phase 2 community pharmacist quotes}

\section{Communication and relationships}

I've had a dilemma fairly recently on someone wanting an emergency supply...she was taking something differently from what was recorded on the computer...It was lucky the surgery was open so I could get in touch... She was taking sertraline[anti-depressant medication] and she was taking two times 100 milligrams where in fact it had been reduced. She did initially take that, but it had been reduced...she got a bit confused. (P3)

I had a guy the other day who was overusing his Airomir (salbutamol) inhaler. He was going through one every two weeks and I was saying, "You shouldn't be using that much" because I can tell by looking at him he's not that ill in a sense, you know, he's in his fifties but he wasn't collapsing on the floor with breathing difficulties or anything. So I ended up phoning the doctors and they actually got him in to see the nurse. (P1)

\section{Changes to practice}

Well I think it should become part [of] the pharmacy contract. An emergency supply really is no different from Care of the Chemist, the minor ailments scheme. So the minor ailments scheme attracts a fee and a consultation fee so why could we not have something similar for the emergency supply scenario? (CP20)

It would be nice if we could package up some sort of service across particular boundaries or groups so we [pharmacists and practice staff] all work together and we don't have the confusion for the patient really.[...] Yes more structure and a more robust system that we could all adhere to, which would be patient friendly. (CP7)

reception staff experienced pressure and disruption from such requests. While the systems in place for managing repeat medication seemed to work well for the majority of patients, there were issues faced by a significant minority, which were related to multiple factors. These included: practice opening hours; forgetfulness; process errors; and competing priorities. If patients had not accessed the emergency supply service, many would have stopped taking their medication or accessed urgent care services, which was considered inappropriate by pharmacists and practice staff. Practice staff and GPs recognised the potential for mixed messages about adherence to have an impact on future medicine-taking.

The methodology used in this study brought a number of benefits and inevitable limitations. The multiphased, mixed methods nature of this study involved collation of data from multiple perspectives and provided a holistic view of the provision of emergency supplies of POMs through community pharmacies. Sufficient data were collected in all qualitative phases to reach theoretical saturation. Peer-to-peer interviewing has been shown to enable interviewees to be more open about issues encountered in practice, with interviewers better placed to probe answers using their professional experience. ${ }^{15}$ Any inconsistencies across interviewers were minimised by group training and a review of transcripts by the RA 
and individual interviewer. The experiences of the group of CPs who were involved as researchers in this multiphased study are published in a separate paper. ${ }^{19}$ This paper provides more detail about the methodological training and data collection techniques provided to the group. CPs' existing rapport with their local general practice team also enhanced the feedback sessions in phase 4 , with open dialogue giving greater understanding of the practice team perspective. However, data were not routinely collected in phase 1 regarding requests for supplies that were refused, and it is not known how many patients were referred to other services to obtain medicines. Moreover, no patient interviews undertaken in phase 3 of the study involved requests for emergency supply of POMs that had been refused. Additionally, the time of request was not recorded, so it is not possible to determine the activity in the 'out-of-hours' period other than at weekends. The response rate among patients was disappointing, and it is likely that there was a self-selection bias, although the impact of this on the data is unclear.

The frequency and characteristics of emergency supply requests were broadly similar to those found in the 1998 study by O'Neill et al. ${ }^{3}$ Comparisons with Health and Social Care Information Centre (HSCIC) data $^{17}$ on prescriptions dispensed in the community suggest that cardiovascular, endocrine and gastrointestinal medicines were requested in proportions that broadly reflect their prescribed usages. However, medicines for respiratory conditions were over-represented among the requests, with $13 \%$ of requests being from this category, when they only account for $6 \%$ of prescribed items nationwide. In line with previous general studies of medicine dispensing, ${ }^{4-7}$ pharmacists and practice staff struggled with the issue of what constituted 'immediate need'. This study extended the field by examining the patient and GP perspective.

The interactions with patients that arise from emergency supplies provide opportunities for CPs to engage with patients around medicines use and adherence. Changes to current practice were supported by CPs and GPs in this study. One approach would be to formalise the current service, remunerating CPs for the extra work involved. It was felt that such a service would have clear benefits in reducing pressure on other services, providing better structure and support for patients and supporting patients in adhering to their treatment to maximise the benefit from this. It should also include a feedback loop between the pharmacy and practice through which repeated requests, and to ensure any inappropriate requests (eg, bypassing a practice medicines review by going to the pharmacy), can be discussed and joint action taken. Technological advances regarding electronic prescribing and access to electronic patient records might also assist in the effective handling of emergency supply requests, although the impact of these advances is currently unclear.

A uniform service may also reduce patient frustration arising from the current pharmacist-level decision- making regarding whether or not an emergency supply or loan is made. However, this may be seen as reducing professional autonomy, which may impact on clinical outcomes for individual patients. A similar service is already commissioned in Scotland, ${ }^{20}$ and the specification for an English scheme has been developed. ${ }^{14}$ The RPS report Now or Never, ${ }^{11}$ regarding new models of care for pharmacy, has emphasised the opportunity for community pharmacy to become a first point of call for patients, thus reducing pressure on other NHS services. Investment in pharmacy services would be justified by the ensuing efficiency savings. Jointly, general practice and community pharmacy would benefit from discussing ways to improve the repeat prescribing process. This may involve formalisation of the emergency supply or loan process in the short term, coupled with medium-term exploration of pharmacy-based repeat prescription management. These practice changes need to be underpinned by open communication and good relationships between the professions; this study shows that emergency supply is a shared challenge to stimulate positive joint working.

Non-adherence can reduce the benefits of medicines, ${ }^{21}$ leading to therapeutic failure with consequential additional economic costs arising from further treatment needs. It was notable that patients did not necessarily perceive missing doses of their medicines to be a problem, and this should be addressed as appropriate during counselling. Furthermore, the opportunity for patients to circumvent the repeat prescribing process by going straight to the pharmacy and requesting a loan may result in dilemmas and discomfort for GPs and pharmacists alike. It has recently been announced that all community pharmacies in England will audit emergency supply of medicines in 2014/2015 as part of their NHS funding settlement. ${ }^{22}$ Further complementary work to examine the patient pathway up to, and following, an emergency supply would help GPs and pharmacists to implement systems to ensure continuous treatment.

\section{CONCLUSION}

CPs regularly provide emergency supplies to patients who have run out of their repeat medication, including during practice opening hours. This may aid adherence, but there is currently no feedback loop to general practice. Patient care and interprofessional communication may be better served by the introduction of a formally structured and funded NHS emergency supply service from community pharmacies, with ongoing optimisation of repeat prescribing. This could form a more coordinated component of better integrated health and social care pathways, thus ensuring that patients benefit from being able to maintain adherence to their prescribed medicines regime.

Author affiliations

${ }^{1}$ School of Pharmacy and Biomolecular Sciences, Liverpool John Moores University, Liverpool, UK 
${ }^{2}$ Division of Health Research, Lancaster University, Lancaster, UK

${ }^{3}$ Green Line Consulting Ltd, Manchester, UK

${ }^{4}$ School of Pharmacy and Biomedical Sciences, University of Central

Lancashire, Preston, UK

${ }^{5}$ Manchester Pharmacy School, University of Manchester, Manchester, UK

${ }^{6}$ NIHR NW Coast Clinical Research Network, Liverpool, UK

${ }^{7}$ Independent Pharmacist Researcher, Manchester, UK

Acknowledgements The research team would like to thank the pharmacist interviewers for their hard work on this project, and the pharmacists who agreed to be interviewed. This study was developed by the North West (NW) Primary Care Pharmacy Research Group workgroup and was facilitated by the NW Primary Care Research Network (PCRN); this steering group includes academic members from the Region's three Schools of Pharmacy (Liverpool John Moores University, the University of Manchester, and the University of Central Lancashire) and practising community pharmacists. This workgroup is actively involved in building research capacity among community pharmacists from both independent and multiple pharmacy companies in the NW region of England.

Collaborators Clive Moss-Barclay initiated the collaborative project and advised on pharmacy training and policy.

Contributors CWM designed data collection tools, monitored data collection for the whole trial, supervised the research assistant and ran training days, wrote the analysis plan, and drafted and revised the manuscript. He is the guarantor. AJM implemented the study, supervised the research assistant and ran training days, analysed the data, and drafted and revised the manuscript.

ECS undertook fieldwork, supervised pharmacists to collect data, analysed the data and drafted and revised the manuscript. DMA revised data collection tools, monitored data collection and analysis, and revised the draft manuscript. SEW revised data collection tools, monitored data collection and analysis, and revised the draft manuscript. NM created and piloted the audit tool, developed the audit analysis plan, monitored data collection and analysis, and revised the draft manuscript. GBP created and piloted the audit tool, developed the audit analysis plan, monitored data collection and analysis, and revised the draft manuscript. NJG initiated the collaborative project, revised data collection tools, monitored data collection and analysis for the whole trial, and drafted and revised the manuscript. All authors designed the study.

Funding This study was funded by a research grant from Pharmacy Research UK http://www.pharmacyresearchuk.org/

Competing interests CWM, AJM and ECS have support from Pharmacy Research UK for the submitted work; and NJG has support from Liverpool John Moores University for the submitted work. AJM has received locum fees from community pharmacy contractors; NJG has received research funding from Pharmacy Research UK and Community Pharmacy Greater Manchester; and NM and GBP are employees of Boots Pharmacy, all of which organisations might have an interest in the submitted work-in the previous 3 years. CWM, AJM, NJG, DMA, NM and GBP are all pharmacists registered with the General Pharmaceutical Council.

Patient consent Obtained.

Ethics approval Ethical approval was obtained in October 2012 from the West Midlands Black Country National Research Ethics Service Committee (12/WM/0364) and Liverpool John Moores University (LJMU) Ethics Committee (12/PBS/005).

Provenance and peer review Not commissioned; externally peer reviewed.

Data sharing statement No additional data are available.

Open Access This is an Open Access article distributed in accordance with the Creative Commons Attribution Non Commercial (CC BY-NC 4.0) license, which permits others to distribute, remix, adapt, build upon this work noncommercially, and license their derivative works on different terms, provided the original work is properly cited and the use is non-commercial. See: http:// creativecommons.org/licenses/by-nc/4.0/

\section{REFERENCES}

1. Statutory Instruments. The Human Medicines Regulations 2012. No. 1916. London: The Stationery Office, 2012.

2. Royal Pharmaceutical Society. Medicines, ethics and practice the professional guide for pharmacists. Number 37. London, 2013.

3. O'Neill R, Rowley E, Smith F. The emergency supply of prescription-only medicines: a survey of requests to community pharmacists and their views on the procedures. Int $\mathrm{J}$ Pharm Pract 2002;10:77-83.

4. Hibbert D, Rees JA, Smith I. Ethical awareness of community pharmacists. Int J Pharm Pract 2000;8:82-7.

5. Cooper RJ, Bissell P, Wingfield J. Dilemmas in dispensing, problems in practice? Ethical issues and law in UK community pharmacy. Clin Ethics 2007;2:103-8.

6. Chaar BB, Brien J, Krass I. Professional ethics in pharmacy practice: developing a psychometric measure of moral reasoning. Pharm World Sci 2009;31:439-49.

7. Deans Z. Ethics in pharmacy practice, for pharmacy practice research trust. 2010.

8. Shepherd MD. Examination of why some community pharmacists do not provide 72-hour emergency prescription drugs to Medicaid patients when prior authorization is not available. $J$ Manag Care Pharm 2013;19:527-33.

9. Urgent Care 24 (UC24)/NHS England (Merseyside). GP Out of Hours services data: Repeat medication requests 1st September-1st September 2013. Liverpool: UC24, 2013.

10. Department of Health. The NHS belongs to the people: a call to action. London: Department of Health, 2013.

11. Smith J, Picton C, Dayan M. Now or Never: shaping pharmacy for the future. The Report of the Commission on future models of care delivered through pharmacy. Royal Pharmaceutical Society, London, 2013

12. Department of Health, Prime Minister's Office. Prime Minister announces $£ 500$ million to relieve pressures on A\&E. https://www. gov.uk/government/news/prime-minister-announces-500-million-torelieve-pressures-on-ae (accessed 1 Oct 2014).

13. Pharmaceutical Services Negotiating Committee (PSNC). New Directions allow Enhanced services for emergency supply. http:// psnc.org.uk/our-news/new-directions-allow-enhanced-services-foremergency-supply/ (accessed 1 Oct 2014).

14. Pharmaceutical Services Negotiating Committee (PSNC). NHS Community Pharmacy Contractual Framework: Enhanced ServiceNHS Emergency Supply Service (at the request of the patient) Draft service specification [Internet]. 2009. http://psnc.org.uk/wp-content/ uploads/2013/07/NHS_emergency_supply_framework.doc (accessed 1 Oct 2014).

15. Chew-Graham CA, May CR, Perry MS. Qualitative research and the problem of judgement: lessons from interviewing fellow professionals. Fam Pract 2002;19:285-9.

16. Hsieh H-F, Shannon SE. Three approaches to content analysis. Qual Health Res 2005;15:1277-88.

17. Prescribing and Primary Care Services: Health and Social Care Information Centre. Prescriptions Dispensed in the Community: England 2002-12. London, 2013.

18. Department of Health. The National Health Service Act 2006. The Pharmaceutical Services (Advanced and Enhanced Services) (England) (Amendment) (No. 2) Directions 2013. London: Department of Health, 2013.

19. Morecroft CW, Gray NJ, Mackridge AJ, et al. Involving community pharmacists in pharmacy practice research: experiences of peer interviewing. Int J Clin Pharm 2015;37:31-5.

20. NHS Scotland. National patient group direction: urgent provision of repeat medicines, appliances and ACBS products. Operational Procedure for Pharmacists. 2013.

21. National Institute for Health and Clinical Excellence (NICE). Clinical guideline 76, Medicines adherence: Involving patients in decisions about prescribed medicines and supporting adherence. London, 2009.

22. Pharmaceutical Services Negotiating Committee (PSNC). PSNC Announcement: Community Pharmacy Funding Settlement for 2014/ 15. PSNC website news, 22nd September 2014. http://psnc.org.uk/ our-news/psnc-announcement-community-pharmacy-fundingsettlement-201415/ (accessed 1 Oct 2014). 